\title{
Effects of the Size of the Measured Surface on the Performance of an Air Cone-jet Sensor for In-process Inspection
}

\author{
Tuqiang Xie, Qingping Yang, Barry E. Jones, Haizhuang Kang, Clive Butler \\ Department of Manufacturing \& Engineering Systems, Brunel University \\ Uxbridge, Middlesex, UB8 3PH, UK; E-mail: empgttx@brunel.ac.uk
}

\begin{abstract}
This paper investigates the effects of the size of the measured surface on the performance of an air-jet sensor using 2-D finite element method. The modeling and experimental results have shown that in the measuring range of $1.5 \mathrm{~mm}$ to $4.5 \mathrm{~mm}$ with a nozzle of diameter of $6 \mathrm{~mm}$, the output of the cone-jet is not significantly affected by the size change from $10 \mathrm{~mm}$ to $14 \mathrm{~mm}$. It also proved that this particular sensor is not suitable for measuring an object with a size less than 9 $m m$.
\end{abstract}

\section{Introduction}

In-process inspection systems for turning centers (lathes) have been under investigations for long time [1]. They are typically based upon an accurate displacement sensor adjacent to the cutting point to provide information for automatic tool condition compensation and control. A novel displacement probe has been recently proposed, which consists of a primary cone-jet sensor [2] and a secondary optic fiber sensor.

The cone-jet sensor is used to measure the clearance between the measured object and the sensor, as shown in Fig. 1. Fluid from a constant pressure supply passes through an annular passage and is directed into a conical jet through a suitably designed nozzle. If the sensor is positioned normal to a plane surface of measured object, the fixed flow from the conical jet is restricted by the measured object and the restriction is varied by moving sensor or object to change the clearance between them. This causes the pressure inside the cone to rise with decreasing clearance. Thus a pressure-measuring device connected to the cone-jet can be calibrated to read clearance $x_{i}$ [3]. The pressure in the stagnant region contained within the flow cone is measured by means of a static tapping. It can be imagined that the different sizes of the objects will have different restrictions to the air flow. Due to the fact that the measured object is irregular with arbitrary size in many inprocess inspections, it is a problem whether the output pressure and sensor performance will depend upon the size of the measured object, which will be investigated in this paper.

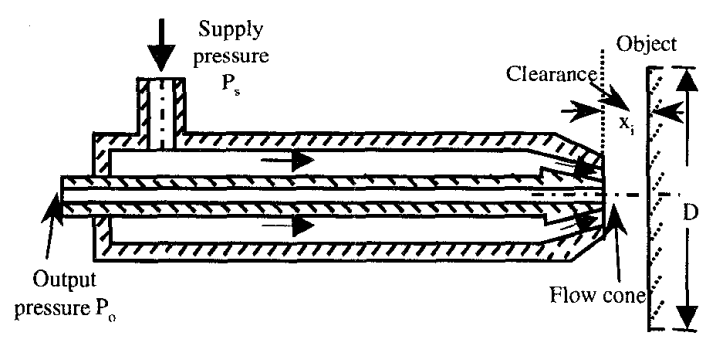

Fig. 1 The cone-jet sensor used to measure the clearance

\section{Finite Element Modeling}

In the fluid analysis of the air cone-jet sensor, the velocity as the boundary condition is zero on the surface of the object, thus size and dimensions of the measured object will determine the boundary conditions restricting the flow. If the theoretical relationship between the clearance and the output pressure can be obtained, this effect can also be deduced from theoretical model and compensated for. However, it is very difficult to establish the theoretical model of the cone-jet sensor due to its complex structure. Here, the finite element method has been used to analyze the effect of the size of the measured object on the measurement [4].

According to fluid dynamics, the flow of a gas may be considered to be incompressible for Mach numbers of 0.3 or less [4]. Since the fluid velocity in the sensor is much less than the sonic velocity, the air flow may be considered 
to be incompressible. The incompressible Navier-Stokes equations are the momentum equations subject to the incompressibility constraint. For simplicity, body force terms are not corsidered. A simple linear constitutive relationship between stress and the rate of straip is assumed. The ecuations on a domain, $\Omega$, are usulally written as:

$$
\begin{aligned}
& \rho\left(\frac{\delta v}{\delta t}+v \cdot \nabla v\right)+\nabla p-\mu \nabla^{2} v=0 \\
& \nabla \cdot v=0
\end{aligned}
$$

where $v$ is velociiy of fluid flow, $p$ the pressure, $f$ the density, $\mu$ the viscosity [6] and $\nabla$ the vector operato.

The numerical simulation of the velocity-pressure formulation requires the discretization of the governing equations. With the advent of digital computers, nany numerical methods are available to convert the governing partial differential equations to a set of algebraic equations (steady-state analysis) and a set of ordinary differential equations (transient analysis). The finite element analysis is a general weighted residual method because it can be applied to both steady-state and transient proolems, linear and nonlinear, involving intricate geometrics.

Currently, two techniques are popular for the simulation of the velocity-pressure: formulation. The first technique uses mixed methods which involve solving for both velocity and pressure. The second is a penalty method, in which the pressure is eliminated by using the slightly compressible form of the incompressibility constraint. Since the penalty method is well understood and easy to implement, it is popular from an application point of view. The incompressibility constraint $\nabla \bullet v=0$ is eliminated by using a penalty parameter. Only the velocity components need to be solved. The pressure can be evaluated as follows:

$$
p=-\lambda \nabla \cdot v
$$

where $\lambda$ is the penalty parameter. It is convenient to compute the pressure at the element centroid and then use a least-square smoothing process to evaluate it at the velocity nodes for post-processing.

Because of its complex structure and the turbulent flow, meshing is difficult and the number of elernents becomes huge when trying to solve in three dimensions. Furthermore, the calculation is also difficult to converge. Theretore, the model is analyzed using two- dimensional finite elements to simplify the calculation and this consideration is reasonable since the cone-jet sensor has a centric symmetry. The meshed model is shown in Fig. 2 with the nozzle diameter of the cone-jet sensor 6 $\mathrm{mm}$. Given the different sizes of the measured object, the different models can be analyzed for different clearances with the fixed air supply source and the corresponding output pressures calculated.

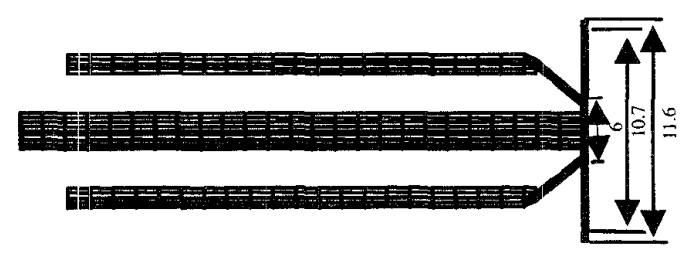

Fig. 2 The meshed model with nozzle diameter of $6 \mathrm{~mm}$

\section{Experiment}

The experimental set-up is shown in Fig.3, a micrometer device is used to move the object step by step. The pressure meter is used to measure the output pressure at the center of the stagnant region of cone-jet sensor. During the experiment, the air flow source provides a constant supply pressure and the air flow is monitored by an air flow meter. The surfaces of the measured objects are circular and have the same finish. The diameters of the objects are $8,9,10,11,12,13$ and $14 \mathrm{~mm}$ and the cone-jet sensor is positioned normal to the measured surface and concentric with the axis of the measured object.

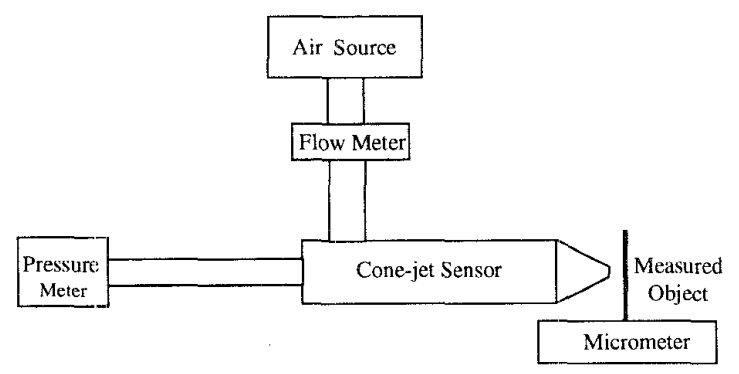

Fig. 3 The diagram of experimental set-up

\section{Results and Discussions}

Given the boundary conditions including the air velocity $v_{s}$ from the supply source, fixed boundary on the interface of air and solid, and free boundary, the model was meshed for certain clearance $x_{i}$. Its output pressure $p_{0}$ 
can be calculated using finite-element method in the fluid flow field. The modeling results are shown in Fig. 4 , corresponding to clearances $x_{i}$ of 1.6 to $4.6 \mathrm{~mm}$. The diameter of the measured object is $10.7 \mathrm{~mm}$ and $11.6 \mathrm{~mm}$, respectively, with other conditions unchanged. The results in Fig. 4 indicated that the output of the cone-jet sensor do not change significantly with the diameter of the measured object within the measurement range of $1.6 \mathrm{~mm}$ to $4.6 \mathrm{~mm}$.

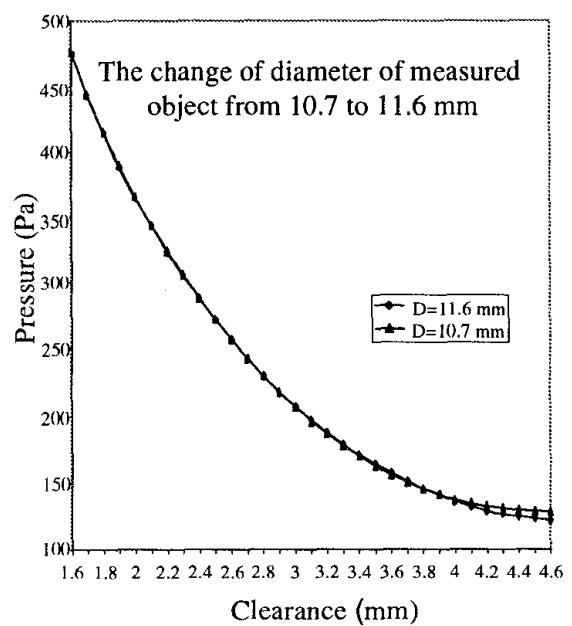

\section{Fig. 4 The modeled results when the diameter of measured object is $\mathbf{1 1 . 6}$ and $10.7 \mathrm{~mm}$}

Fig. 5 gives the modeling results when the size of the measured object decreases from 10.7 to $8 \mathrm{~mm}$. In the measurement range of 1.6 to $3.2 \mathrm{~mm}$, the effect of the diameter of the measured object on the output pressures for the same clearance very small, but above $3.2 \mathrm{~mm}$ it becomes more significant with the output pressure decreasing with the diameter of measured object. When the diameter of the measured object is further decreased from 8 to $5.3 \mathrm{~mm}$, the modeling results are shown in Fig. 6. It is obvious that the output pressures would seriously be influenced by the size of measured object.

During the experiments, when the clearance $x_{i}$ was changed from $0.6 \mathrm{~mm}$ to $4.5 \mathrm{~mm}$, the air flow as measured by the flow meter (shown in Fig. 3) was kept at 37.5 liters/minute. The three objects of different diameters (i.e. 12,13 and $14 \mathrm{~mm}$ ) were measured and the experimental results are shown in Fig. 7. It shows that the output pressure is a function of the clearance and the function does not change with the diameter of the measured object. Comparing Fig. 4 with Fig. 7, the relationships between the pressure and clearance have

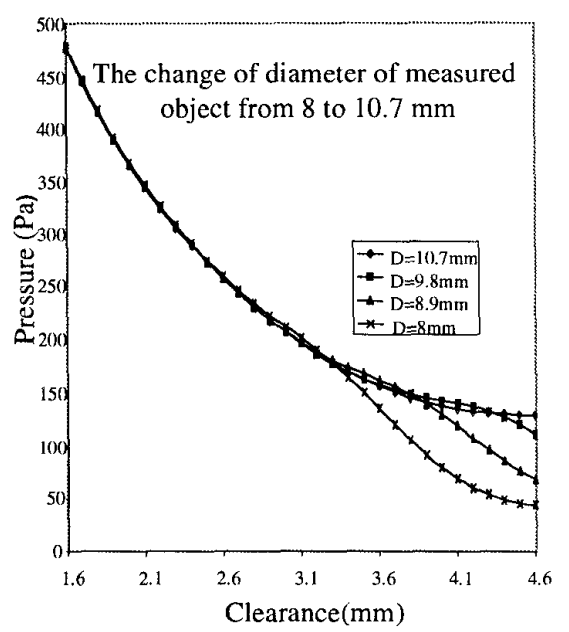

Fig. 5 The modeled results when the diameter of measured object is $10.7,9.8,8.9$ and $8 \mathrm{~mm}$ respectively

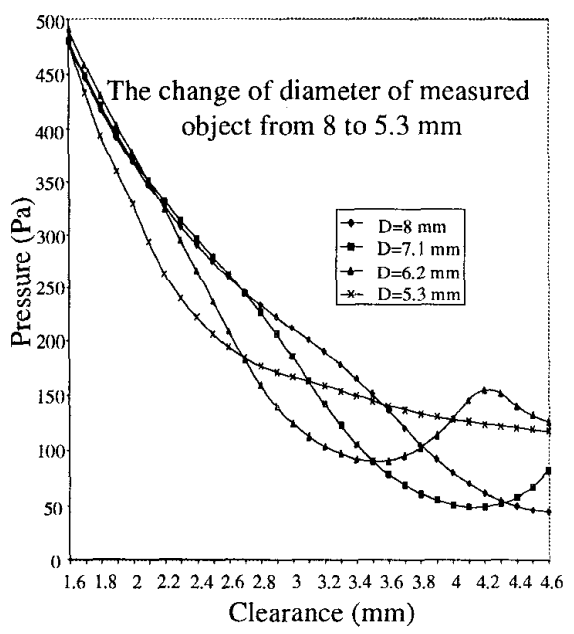

Fig. 6 The modeled results when the diameter of measured object changed from 8 to $5.3 \mathrm{~mm}$.

similar patterns, although the experimental output pressure is higher than that obtained from the modeling for the same clearance. This is due to different boundary conditions, 2-D finite-element approximation and meshing selection. Objects with smaller diameters $(8,9,10,11 \mathrm{~mm})$ were also measured, with the experimental results shown in Fig. 8. It is obvious that output pressure values present peaks when the diameter of the measured object is 8 and 9 $\mathrm{mm}$ and clearance below $1.7 \mathrm{~mm}$. Even in the range of 1.7 to $4.5 \mathrm{~mm}$, there exists generally an offset of the output pressure when the diameter is $8 \mathrm{~mm}$. In Fig. 8 , it also shows that the diameters above $10 \mathrm{~mm}$ do not affect the 
measurement of the cone-jet sensor in the range of 1.5 to $4.5 \mathrm{~mm}$.

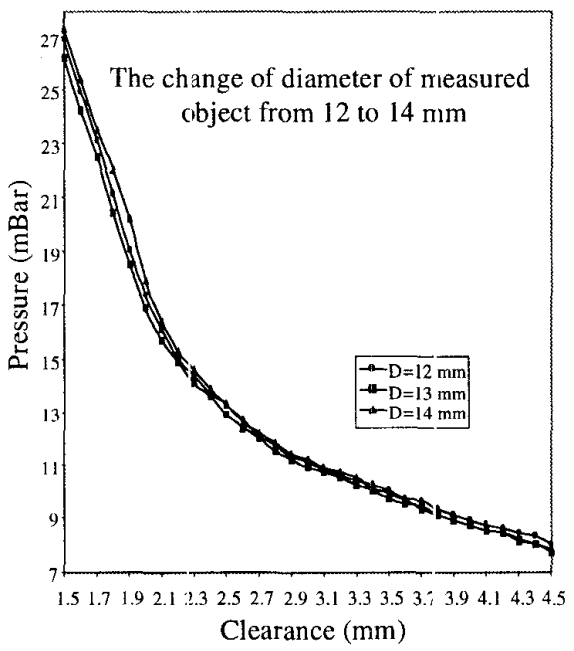

Fig. 7 The experinaental results when diameters of measured object changed from 8 to $11 \mathrm{~mm}$.

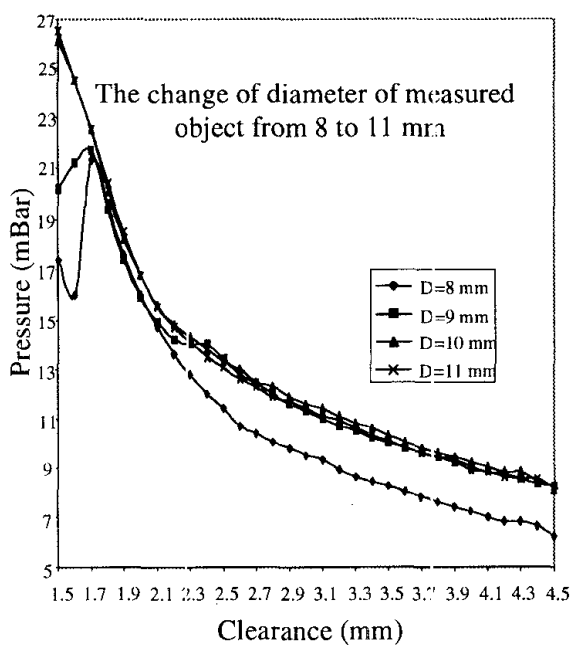

Fig. 8 The experimental results when diameters of: measured object changed from 12 to $14 \mathrm{~mm}$.

\section{Conclusions}

Because of the complex geometry and boundary conditions of the cone-jet sensor, it is very difficult to construct an analytical solution. The $2 \cdots \mathrm{D}$ finite-element method has been used to analysis the effect of the size of the measured object on clearance measurement and provided a guidance for experiment. The modeling and experimental results indicated that the variation in the size of the measured object has little effect on measurement in the range of 1.5 to $4.5 \mathrm{~mm}$, if the size large enough (above $10 \mathrm{~mm}$ for a nozzle with a diameter of $6 \mathrm{~mm}$ ). With a smaller measured object (below $10 \mathrm{~mm}$ in this case) the measurement is seriously affected by the variation in the size of the measured object. More results will be reported in the future.

\section{References}

1. S. D. Murphy, In-Process Measurement and Control, Marcel Dekker, INC (New York), 1990.

2. L. C. Hall, B. E. Jones, "An investigation into the use of a cone-jet sensor for clearance and eccentricity measurement in turbomachinery", Proc Instn Mech Engrs Vol 190, 17/76, pp.23-30.

3. T. Q. Xie, Q. Yang, Y. J. Au and B. E. Jones, Modelling of an Air Cone-jet Sensor using Finite Element Method, EUROSENSORS XII, Southampton, 13-16 September 1998, pp1159-1162.

4. G. F. Carey, J. T. Oden, Finite elements: Fluid Mechanics, Prentice-Hall (New Jersey), 1986 